\title{
DIE RECHTSSPRECHUNG DES VERFASSUNGSGERICHTSHOFS IN BEZUG AUF DIE MEINUNGSFREIHEIT
}

DOI: http://dx.doi.org/10.12775/TSP-W.2020.006

Date of receipt: 25.04 .2020

Date of acceptance: 15.09.2020

The jurisdiction of the Polish Constitutional Court regarding freedom of expression - Freedom of expression is a basic human right. The existence of freedom of expression in the state is the foundation of democracy. The source of freedom of expression is the dignity of the human person. The role of constitutional regulations boils down to confirming the existence of this freedom. However, the jurisprudence of the Constitutional Tribunal emphasizes that freedom of expression is not absolute.

Keywords: jurisprudence, freedom of expression, dignity.

Wolność wypowiedzi w świetle orzecznictwa Trybunału Konstytucyjnego RP. Wolność wypowiedzi to podstawowe prawo człowieka. Istnienie w państwie swobody wypowiedzi jest fundamentem demokracji. Źródłem wolności wypowiedzi jest godność osoby ludzkiej. Rola unormowań konstytucyjnych sprowadza się do potwierdzenia istnienia tej wolności. W orzecznictwie Trybunału Konstytucyjnego podkreśla się jednak, że wolność wypowiedzi nie ma charakteru absolutnego.

Słowa kluczowe: orzecznictwo, wolność słowa, godność.

\footnotetext{
${ }^{1}$ Magdalena Lis - Ph.D. Student, Department of Constitutional Law, Faculty of Law and Administration, University of Łódź, ORCID 0000-0003-4197-1404, e-mail: magdalena.suska@ wpia.uni.lodz.pl.
} 
1. Die Meinungsfreiheit gilt in den modernen Demokratien als ein grundlegendes Menschenrecht, und dadurch auch als der Kern des demokratischen politischen Systems. Diese Freiheit, seine Meinung ausdrücken zu können, stellt den Gegenstand zahlreicher wissenschaftlicher Forschungen dar. Obwohl jedoch das wissenschaftliche Werk auf diesem Gebiet bedeutend ist, bestehen weiterhin zahlreiche Fragen, die Anlass für weitere Diskussionen bilden. Auch die Rechtssprechung von Gerichten entscheidet über neue Probleme im Zusammenhang mit der Meinungsfreiheit. Eine besonders wichtige Rolle spielt hier der Verfassungsgerichtshof [Trybunat Konstytucyjny], der über der Verfassungsmäßigkeit der Gesetze wacht und deren Pflicht es ist, auf eventuelle Abweichungen zu reagieren. Die Meinungsfreiheit kann nicht ohne den Zusammenhang mit ihren Einschränkungen analysiert werden. Erst, wenn man die Grenzen der Meinungsfreiheit - die den tatsächlichen Ausmaß der Freiheit eines Einzelnen bestimmen - kennengelernt hat, kann man das Thema der Meinungsfreiheit behandeln. Zutreffend ist also die Bemerkung, dass das Verfassungsgericht den endgültigen Garanten der Rechte und Freiheiten darstellt ${ }^{2}$.

Zweck dieses Artikels ist eine Darstellung des Problems der Meinungsfreiheit auf der Grundlage der Rechtssprechung des Verfassungsgerichtshofs. Es ist vor allem erforderlich, die Grenzen dieser Freiheit zu bestimmen, ebenso wie die Voraussetzungen und die Art und Weise, wie diese Einschränkung erfolgt. In Bezug auf die Klausel des demokratischen Rechtsstaats basierend ist es von wesentlicher Bedeutung, die Intensität der Einschränkung der besprochenen Freiheit festzulegen. Unter der Berücksichtigung dieses Aspekts ist eine Analyse der Rechtssprechung des Verfassungsgerichtshofs erforderlich, um die Aurea Mediocritas zu finden.

2. Wenn man an die „Meinungsfreiheit“ denkt, muss dabei berücksichtigt werden, dass es sich um einen komplexe Erscheinung handelt. Sie bildet eine Ableitung der Gedankenfreiheit, die einen absoluten und vorbehaltslosen Charakter besitzt. Die Frage nach der Meinungsfreiheit darf nicht auf eine Kategorie sensu stricto beschränkt werden. Sie umfasst sämtliche Arten der Äußerung eigener Gedanken anderen Personen gegenüber. Die Form der geäußerten Meinungen hat dabei keinerlei Bedeutung, weil dies - wie der Verfassungsgerichtshof mit Recht festgestellt hat - sowohl in Worten (mündlich und schriftlich), als auch durch Bilder oder entsprechende Kleidung und Abzeichen erfolgen kann³.

${ }^{2}$ M. Safjan, Trybunat Konstytucyjny po trzydziestu latach - doświadczenie i przyszłość, Przegląd Konstytucyjny 2017, Nr. 1, S. 39.

${ }^{3}$ Urteil des Verfassungsgerichtshofs vom 20.07.2011, Aktenzeichen K 9/11, OTK ZU 6A/2011, Pos. 61. Siehe: 1) J. Sobczak, Wolność ekspresji artystycznej. Standardy europejskie i rzeczywistość polska, [in:] Rada Europy a przemiany demokratyczne w państwach Europy Środ- 
Die Ursprünge der Meinungsfreiheit liegen in der Würde des Menschen, die angeboren ist ${ }^{4}$. Unter Berücksichtigung von Art. 30 der Verfassung der Republik Polen verleiht das Grundgesetz der menschlichen Würde den Charakter eines aus der Verfassung abgeleiteten Werts mit einer grundlegenden Bedeutung, unter deren Gesichtspunkt die Auslegung der Bestimmungen der Verfassung der Republik Polen zu erfolgen hat. Es ist die Würde eines Menschen, die die Grundlage seiner Freiheiten und Rechte bildet. Gleichzeitig bestimmt die polnische Verfassung in Art. 30, dass die Würde angeboren und unverzichtbar ist, und dass die öffentlichen Organe dazu verpflichtet sind, diese zu achten und - vor allem - zu schützen ${ }^{5}$. Wie der Verfassungsgerichtshof urteilte, ,,bildet die Meinungsfreiheit eines der grundlegenden Menschenrechte, die auf eine natürliche Weise jedem Menschen zustehen"'. Damit beschränkt sich die Rolle der verfassungsrechtlichen Regelungen auf eine Bestätigung, dass diese Freiheit existiert, sowie auf die Bestimmung der wichtigsten Aspekte dieser Freiheit und auf die Festlegung der notwendigen Garantien für die erforderlichen Einschränkungen ${ }^{7}$. Unter Berücksichtigung von Art. 2 der polnischen Verfassung hat der Verfassungsgerichtshof korrekt festgestellt, dass die Rolle des Gesetzgebers unter anderem darin besteht, solche gesetzlichen Regelungen zu treffen, die sich positiv auf den Zustand der Unsicherheit auswirken. Wenn man hingegen auf die Axiologie der Verfassung zurückgreift, die auf dem Fundament der angeborenen und unverzichtbaren menschlichen Würde basiert, verpflichtet diese den Gesetzgeber dazu, solche Gesetze zu schaffen, die auf das Wohlergehen des Menschen ausgerichtet sind ${ }^{8}$. Daraus folgt, dass der demokratische Rechtsstaat ein Staat ist, der auf der Ach-

kowej i Wschodniej w latach 1989-2009, Hrsg. J. Jaskiernia, Toruń 2010, S. 612-639; 2) Urteil des EGMR vom 24.05.1988 in der Sache Muller und andere gegen die Schweiz (Klage Nr. 10737/84).

${ }^{4}$ Siehe: 1) Urteil des Verfassungsgerichtshofs vom 15.10.2002, Aktenzeichen Sk 6/02, OTK ZU 5A/2002, Pos. 65; 2) Urteil des Verfassungsgerichtshofs vom 30.09.2008, Aktenzeichen K 44/07, OTK ZU 7A/2008, Pos. 126; 3) Urteil des Verfassungsgerichtshofs vom 09.07.2009, Aktenzeichen Sk 48/05, OTK ZU 7A/2009, Pos. 108; 4) Beschluss des Verfassungsgerichtshofs vom 22.07.2015, Aktenzeichen Sk 20/14, OTK ZU 7A/2015, Pos. 115; 5) M. Piechowiak, Prawo naturalne a prawa człowieka, [in:] Substancja. Natura. Prawo naturalne. Zadania wspótczesnej metafizyki, Hrsg. A. Maryniarczyk, K. Stępień, P. Gondek, Lublin 2006, s. 391-409; 6) M. Piechowiak, Filozofia praw człowieka. Prawa człowieka w świetle ich międzynarodowej ochrony, Lublin 1999.

${ }^{5}$ Urteil des Verfassungsgerichtshofs vom 22.01.2013, Aktenzeichen P 46/09, OTK ZU 1A/2013, Pos. 3.

${ }^{6}$ Beschluss des Verfassungsgerichtshofs vom 02.03.1994, Aktenzeichen W 3/93, OTK ZU 1994, Pos. 17.

${ }^{7}$ Siehe: Urteil des Verfassungsgerichtshofs vom 18.02.2004, Aktenzeichen P 21/02, OTK ZU 2A/2004, Pos. 9.

${ }^{8}$ Urteil des Verfassungsgerichtshofs vom 19.06.2012, Aktenzeichen P 41/10, OTK ZU 6A/2012, Pos. 65. 
tung des Menschen basiert. Die staatliche Anerkennung, dass die individuelle Person ein selbstständiges Subjekt darstellt, das dazu imstande ist, seine Vorgehensweise selbst zu bestimmen, sollte dieser Person eine Freiheit zusprechen, die die Conditio sine qua non der Selbstentwicklung darstellt. Die Meinungsfreiheit, von der in Art. 54 Abs. 1 der Verfassung der Republik Polen die Rede ist, bildet unter anderem ein Element des Inhalts des Freiheitsprinzips einer individuellen Person, im Zusammenhang mit deren angeborenen und unverzichtbaren Würde. Der Verfassungsgeber hat darüber entschieden, dass ,,jedem die Freiheit der Äußerung seiner Meinungen sowie der Gewinnung und Verbreitung von Informationen garantiert wird“. In der genannten Vorschrift bestimmt die Verfassung der Republik Polen drei separate Freiheiten, die miteinander verbunden und interdependent sind, und zwar: die Freiheit der Äußerung seiner Ansichten, die Freiheit der Gewinnung und der Verbreitung von Informationen'. Über die Aussagefreiheit ist in Kapitel II. der Verfassung der Republik Polen mit dem Titel „Freiheiten, Rechte und Pflichten des Menschen und des Staatsbürgers“ die Rede - im Katalog der persönlichen Freiheiten und Rechte, die also mit der Person des Menschen und Staatsbürgers strikt verbunden sind. Dies entscheidet darüber, dass der „Meinungsäußerungsfreiheit“" nicht ausschließlich der Charakter einer politischen Freiheit zugeschrieben werden kann, womit diese Freiheit in der öffentlichen Sphäre hauptsächlich assoziiert wird (insbesondere in den Social Media). In der Rechtssprechung des Verfassungsgerichtshofs werden zwei Ebenen der Meinungsäußerungsfreiheit unterschieden, also 1) im Sinne der individuellen Rechte des Menschen und Staatsbürgers in der Privatsphäre, und 2) in der öffentlichen und politischen Sphäre. Die Meinungsfreiheit hat also einen gemischten Charakter $^{10}$, weil sie nicht auf eine Teilnahme an einem der mög-

${ }^{9}$ Urteil des Verfassungsgerichtshofs vom 20.02.2007, Aktenzeichen P 1/06, OTK ZU 2A/2007, Pos. 11. In diesem Beschluss erläutert der Verfassungsgerichtshof den Unterschied zwischen der Gewinnung und der Verbreitung von Informationen: „Die Freiheit der Gewinnung von Informationen, von der in Art. 54 Abs. 1 der Verfassung die Rede ist, ist ein umfangreicherer Begriff, als das Recht auf die Gewinnung von Informationen (Art. 61 der Verfassung). Sie umfasst auch die Freiheit der Suche nach Informationen, die insbesondere für diejenigen wichtig ist, die über die sozialen Kommunikationsmittel verfügen. Die Freiheit der Verbreitung von Informationen bedeutet sowohl, dass die gesammelten Informationen durch die diese veröffentlichende Person individuell ausgewählten Institutionen zur Verfügung gestellt werden, als auch, dass die Information veröffentlicht wird, diese also öffentlich zur Kenntnis gegeben wird, also an nichtindividualisierte Empfänger, insbesondere durch Massenmedien. Die Freiheit der Verbreitung von Informationen bezüglich des Privatlebens verfügt über bedeutend weiter gesetzte Einschränkungen, als die Freiheit der Gewinnung von Informationen".

${ }^{10}$ Urteil des Verfassungsgerichtshofs, K 9/11. 
lichen Diskurse (insbesondere des politischen) beschränkt werden kann ${ }^{11}$. Die Meinungsfreiheit ist auch mit ausführlicheren Rechten verbunden, wie z.B. mit der Freiheit der Kommunikation (Art. 49 der Verfassung der Republik Polen), dem Recht auf Informationen über die Tätigkeit der Organe der öffentlichen Gewalt sowie der öffentliche Ämter bekleidenden Personen (Art. 61 der Verfassung der Republik Polen), oder etwa dem Recht auf Informationen über den Zustand und Schutz der Umwelt (Art. 74, Abs. 3 der Verfassung). Letztendlich hat der Verfassungsgerichtshof auch anerkannt, dass die Meinungsfreiheit und die Pressefreiheit einen komplementären Charakter besitzen ${ }^{12}$. Es muss die Tatsache betont werden, dass der Umfang der Meinungsfreiheit auch durch völkerrechtliche Vorschriften bestimmt wird. Von Bedeutung ist hier unter anderem Art. 10 Abs. 1 der Konvention zum Schutze der Menschenrechte und der Grundfreiheiten ${ }^{13}$, die am 4. November 1959 verfasst und durch Polen am 15. Dezember 1992 ratifiziert worden ist. Gemäß der Bestimmung aus Abs. 1 ,hat jeder das Recht auf eine freie Meinungsäußerung“, und es wird präzisiert, dass „dieses Recht die Freiheit auf eine eigene Meinung sowie auf das Erhalten und Übermitteln von Informationen und Ideen ohne eines Eingriffs der öffentlichen Behörden und ohne Beachtung der Staatsgrenzen umfasst". Die völkerrechtlichen Regelungen bestimmen die allgemeinen Rahmen, mit denen die gesetzlichen Regelungen der untergeordneten Institutionen übereinstimmen sollten. Dies bedeutet, dass polnische gesetzliche Regelungen, insbesondere das Grundgesetz, die MeinungsäuBerungsfreiheit auf einem Niveau garantieren sollten, das mit dem Niveau in den völkerrechtlichen Menschenrechte vergleichbar ist. Der Verfassungsgerichtshof

11 1) Urteil des Verfassungsgerichtshofs vom 15.05.2008, Aktenzeichen Sk 43/05, OTK ZU 4A/2008, Pos. 57; 2) Urteil des Verfassungsgerichtshofs vom 14.12.2011, Aktenzeichen Sk 42/09, OTK ZU 10A/2011, Pos. 118.

${ }^{12}$ Urteil des Verfassungsgerichtshofs, Sk 43/05.

${ }^{13}$ Die in Rom am 4. November 1950 verfasste Konvention zum Schutze der Menschenrechte und der Grundfreiheiten, später geändert durch die Protokolle Nr. 3, 5 und 8 und ergänzt durch das Protokoll Nr. 2 (GBl. [Dz.U.] von 1993, Nr. 61, Pos. 284, mit Änderungen). Andere völkerrechtliche Dokumente in Bezug auf die Frage der Meinungsäußerungsfreiheit: 1) die Allgemeine Erklärung der Menschenrechte vom 10.12.1948 - Art. 19, der besagt: ,Jeder hat das Recht auf Meinungsfreiheit und freie Meinungsäußerung; dieses Recht schließt die Freiheit ein, Meinungen ungehindert anzuhängen sowie über Medien jeder Art und ohne Rücksicht auf Grenzen Informationen und Gedankengut zu suchen, zu empfangen und zu verbreiten; 2) der Internationale Pakt über bürgerliche und politische Rechte vom 16.12.1966 - Art. 13; 3) die Charta der Grundrechte der Europäischen Union vom 7.12.2000 - Art. 11 Abs. 1: „Jede Person hat das Recht auf Gedanken-, Gewissens- und Religionsfreiheit. Dieses Recht umfasst die Freiheit, die Religion oder Weltanschauung zu wechseln, und die Freiheit, seine Religion oder Weltanschauung einzeln oder gemeinsam mit anderen öffentlich oder privat durch Gottesdienst, Unterricht, Bräuche und Riten zu bekennen“. 
hingegen ist der Meinung, dass ,,[der Verfassungsgerichtshof - M.L.] (...) keine Möglichkeiten erkennt, die Geltungskraft einer Verfassungsnorm Allen durch die Tatsache in Frage zu stellen, dass eine gegensätzliche gemeinschaftliche Regelung in das System des europäischen Rechts eingeführt worden ist“. Und weiter: „Die Verfassungsnormen auf dem Gebiet der Rechte und Freiheiten einer individuellen Person bestimmen die minimale und unüberschreitbare Schwelle, die infolge einer Einführung von gemeinschaftlichen Regelungen weder herabgesetzt, noch in Frage gestellt werden kann. Diesbezüglich erfüllt die Verfassung ihre garantierende Rolle unter dem Gesichtspunkt des Schutzes von Rechten und Freiheiten, die deutlich in ihr bestimmt sind, und zwar im Verhältnis zu allen Subjekten, die auf dem Gebiet ihrer Anwendung aktiv sind. Die «Europarechtfreundliche» Auslegung hat ihre Grenzen. Sie darf in keinerlei Situation dazu führen, dass Resultate entstehen, die im Widerspruch zum deutlichen Wortlaut der verfassungsrechtlichen Regelungen stehen und die nicht mit einem Minimum der durch die Verfassung ausgedrückten Garantiefunktionen in Übereinstimmung gebracht werden können ${ }^{14 ،}$.

Die Regelung der Frage der Meinungsäußerungsfreiheit im allerhöchsten Rechtsakt in Polen wurde also bestätigt, es ist jedoch notwendig, den Umfang des entsprechenden Schutzes der Meinungsfreiheit zu bestimmen. Ipso facto der erwähnten Rechtssprechung des Verfassungsgerichtshofs, bestätigt dies die Existenz eines Pluralismus der Meinungsäußerung. In Art. 54 Abs. 1 der polnischen Verfassung hat der Verfassungsgeber angenommen, dass ,,jedem” die Meinungsfreiheit garantiert wird. Der Begriff ,,jeder" muss hier im weiteren Sinne verstanden werden: die Meinungsfreiheit wird jedem Subjekt garantiert, das der Jurisdiktion des polnischen Staates untersteht, unabhängig etwa von dessen Rasse, Hautfarbe, sexuelle Orientierung oder Vermögenssituation. Der Verfassungsgerichtshof hat angenommen, dass nicht nur natürliche Personen ein Subjekt der durch die Verfassung der Republik Polen bestimmten Meinungsfreiheit sind, sondern auch Kollektivsubjekte, zu denen auch politische Parteien und Wahlkomitees gezählt werden müssen. Der Verfassungsgerichtshof ging davon aus, dass auch ihnen diese Freiheit zusteht, weil ,[Institutionen - M.L.] schließlich aus natürlichen Personen bestehen, und in deren Namen äußern sie sich ${ }^{15}$ ". In Polen funktionieren im Rechtsverkehr juristische Personen, also Organisationseinheiten, die über keine Rechtsfähigkeit verfügen. Von besonderer Bedeutung ist, dass

${ }^{14}$ Urteil des Verfassungsgerichtshofs vom 11.05.2005, Aktenzeichen K 18/04, OTK ZU 5A/2005, Pos. 49. Siehe: Urteil des Verfassungsgerichtshofs vom 16.11.2011, Aktenzeichen Sk 45/09, OTK ZU 9A/2011, Pos. 97.

${ }^{15}$ Urteil des Verfassungsgerichtshofs, K 9/11. 
man sich darüber im Klaren ist, dass diese Organisationen über keinen Verstand und keine Fähigkeit zur Meinungsäußerung oder Kommunikation verfügen. Diese Organisationen gelten ausschließlich in der juristischen Sphäre als ein Subjekt. Wenn diese Subjekte also die Meinungsfreiheit nutzen, handelt es sich um eine Konsequenz dessen, dass konkrete natürliche Personen diese Freiheit nutzen. Es darf festgestellt werden, dass diese Freiheit dem Menschen zusteht, weil nämlich diese Subjekte der juristischen Verhältnisse über eigene Meinungen verfügen und Informationen gewinnen und verbreiten können. Im juristischen Sinne hingegen können natürliche Personen diese Freiheit im eigenen Namen und auf eigene Verantwortung nutzen, oder aber im Namen und zugunsten von Institutionen. In dieser Frage hat sich der Verfassungsgerichtshof geäußert und festgestellt, dass eine Meinungsäußerung einer natürlichen Person im Namen einer Institution sowieso bedeutet, dass diese Person ihre Meinungsfreiheit nutzt, und damit ist sie nicht nur „der Mund“ dieser Institution ${ }^{16}$.

3. Das Bestehen der Meinungsfreiheit in einem Staat bildet die Fundamente der Demokratie. Im Prinzip ermöglicht eine uneingeschränkte Freiheit der Äußerung der eigenen Meinung einer Person die Suche nach der Wahrheit ${ }^{17}$, die einen kostbaren Wert für das Bestehen eines demokratischen Staates darstellt. Ein Staat, der diese Freiheit nicht achtet, kann nicht als demokratisch gelten. Die Meinungsäußerungsfreiheit ist eine Bedingung für die Selbstverwirklichung des Individuums ist, das nicht nur seine Ansichten gegenüber einer bestimmten Gruppe von Empfängern äußern, sondern auch Informationen gewinnen und diese weitergeben will; dies bezieht sich auch auf das Recht auf die Verbreitung von Ansichten und Meinungen anderer ${ }^{18}$. Wie in der Theorie und der Rechtssprechung mit Recht dargestellt wird, kann die Meinungsäußerung nicht nur auf die Äußerung von positiven oder neutralen Ansichten begrenzt werden, sondern auch solche umfassen, die als empörend angesehen werden ${ }^{19}$. Man kann sogar behaupten, dass es nicht möglich wäre, dass ein demokratischer Staat ohne einen heftigen Meinungsaustausch zwischen Gegnern bestehen würde. A natura rei spielt hier die Toleranz und Offenheit auf Ansichten anderer Diskursteil-

${ }^{16}$ Urteil des Verfassungsgerichtshofs vom 30.10.2006, Aktenzeichen P 10/06, OTK ZU 9A/2006, Pos. 128.

${ }^{17}$ Siehe: A. Biłgorajski, Granice wolności wypowiedzi. Studium konstytucyjne, Warszawa 2013.

${ }_{18}$ Siehe: Urteil des Verfassungsgerichtshofs vom 29.09.2008, Aktenzeichen Sk 52/05, OTK ZU 7A/2008, Pos. 125.

${ }^{19}$ Urteil des Verfassungsgerichtshofs vom 23.03.2006, Aktenzeichen K 4/06, OTK ZU 3A/2006, Pos. 32. Urteil des EGMR vom 23.04.1992 in der Sache Castells v. Spanien (Klage Nr. 11798/85). 
nehmer eine besonders wichtige Rolle. Es kann angenommen werden, dass es sich hier um Grundelemente der Demokratie handelt. Die Aktivität einer Person bildet einen Beitrag zum Aufbau einer Bürgergesellschaft, einschließlich eines bewussten Bürgers. Die Debatte und der Austausch von Meinungen bildet das Gemeinwohl. Natürlich ist die Teilnahme an öffentlichen Kundgebungen oder die Mitgliedschaft in sozialen Organisationen auch eine Form des Engagements am staatlichen Leben, und damit die Sorge um das Gemeinwohl, das durch den Staat und die Form der Meinungsfreiheit gebildet wird. In der Gesellschaft bedeutet die Meinungsäußerungsfreiheit deren Entwicklung und bestimmt über die Reife einer Gesellschaft, und letztendlich über die Transparenz in der öffentlichen Sphäre. Der Diskurs über wichtige Probleme, mit denen die Gesellschaft zu tun hat, ist für die öffentliche Meinung von Bedeutung und drängt zur Reflexion, aber manchmal auch zu einer Reaktion seitens der öffentlichen Gewalt. Dies sollte ausschließlich als ein Vorteil angesehen werden, weil es über eine effektive Funktionsweise der öffentlichen Meinung entscheidet, die auf die öffentlichen Angelegenheiten ausgerichtet ist ${ }^{20}$. Dies kann nicht ohne einen Zusammenhang mit Art. 4 Abs. 1 der Verfassung der Republik Polen betrachtet werden, der besagt, dass , ,die oberste Gewalt in der Republik Polen dem Volk zusteht“. Der Souverän übt seine Gewalt über seine Vertreter oder direkt aus, und die Instrumente, die eine direkte Machtausübung ermöglichen, sind landesweite Referenden oder lokale Volksabstimmungen ${ }^{21}$. Daher ist die Äußerung der eigenen Meinung über Vertreter der Staatsgewalt eine besondere Ausdrucksform der individuellen Rechte und Freiheiten, was auch die Form einer Petition, einer Versammlung oder einer Diskussion in den Social Media annehmen kann. Die Freiheit der Diskussion, auch einer politischen, bildet das Fundament einer demokratischen Ge-

${ }^{20}$ Urteil des Verfassungsgerichtshofs vom 12.02.2015, Aktenzeichen Sk 70/13, OTK ZU 2A/2015, Pos. 14. Zu den Themen, über die in der öffentlichen Sphäre umfangreich diskutiert worden ist, gehören unter anderem: 1) Urteil des Verfassungsgerichtshofs vom 28.05.1997, Aktenzeichen K 26/96, OTK ZU 2/1997, Pos. 19 (über das Pronzip des Schutzes des menschlichen Lebens als eines Verfassungswertes; der Unstimmigkeit des „Antiabtreibungsgesetzes“ mit der Kleinen Verfassung); 2) Urteil des Verfassungsgerichtshofs vom 01.07.2014, Aktenzeichen Sk 6/12, OTK ZU 7A/2014, Pos. 68 (bezüglich der Höhe der Geldstrafe für die Beseitigung von Bäumen oder Sträuchern ohne die notwendige Erlaubnis); 3) Urteil des Verfassungsgerichtshofs vom 28.10.2015, Aktenzeichen K 21/14, OTK ZU 9A/2015, Pos. 152 (bezüglich des einkommenssteuerfreien Betrags); 4) Urteil des Verfassungsgerichtshofs vom 09.03.2016, Aktenzeichen K 47/15, OTK ZU A/2018, Pos. 31 (bezüglich der Änderung des Gesetzes über den Verfassungsgerichtshof).

${ }^{21}$ Urteil des Verfassungsgerichtshofs vom 20.04.2017, Aktenzeichen K 10/15, OTK ZU A/2017, Pos. 31. Siehe: 1) Urteil des Verfassungsgerichtshofs vom 24.11.2010, Aktenzeichen K 32/09, OTK ZU 9A/2010, Pos. 108; 2) Urteil des Verfassungsgerichtshofs vom 11.08.2016, Aktenzeichen K 39/16, OTK ZU A/2018, Pos. 32. 
sellschaft und nimmt besonders in der Zeit einer Diskussion über die wichtigsten Werte oder Institutionen eine große Bedeutung an ${ }^{22}$. Dabei darf diese Freiheit nicht absolutisiert werden, was der Verfassungsgerichtshof mehrmals in seinen Entscheidungen betont hat, insbesondere wenn es zu einer Verletzung anderer Werte kommen würde, die durch die Verfassung geschützt werden, wie etwa der Würde, der Ehre oder der religiösen Empfindungen ${ }^{23}$. Der Europäische Gerichtshof für Menschenrechte war der Meinung, dass die Meinungsäußerungsfreiheit auch Situationen umfasst, in denen die geäußerten Ansichten die Empfindungen anderer Personen verletzen ${ }^{24}$, trotzdem werden Ausnahmen auf diesem Gebiet zugelassen. Art. 54 der Verfassung der Republik Polen bezieht sich in seinem Inhalt nicht auf Art. 31 Abs. 3 der polnischen Verfassung ${ }^{25}$. In der Rechtssprechung des Verfassungsgerichtshofs wird betont, dass Art. 31 Abs. 3 den Charakter eines allgemeinen Prinzips hat und auch dann zur Anwendung kommt, wenn dies nicht expressis verbis durch die Vorschriften in der Verfassung bestimmt wird. Es kann angenommen werden, dass die Verhältnismäßigkeit der Einschränkungen eine Ergänzung der Norm aus Art. 54 Abs. 1 darstellt ${ }^{26}$. Es ist selbstverständlich, dass die Zulassung durch den Verfassungsgeber einer Einschränkung der Meinungsfreiheit den Anforderungen entsprechen muss, die im Grundgesetz verankert $\operatorname{sind}^{27}$.

Es kann getrost festgestellt werden, dass insbesondere in der polnischen Gesellschaft die Kritik von Ansichten, die bestimmte, von der Mehrheit ange-

${ }^{22}$ Urteil des EGMR vom 08.07.1986 in der Sache Lingens v. Österreich (Klage Nr. 9815/82).

${ }^{23}$ Urteil des Verfassungsgerichtshofs vom 25.11.2008, Aktenzeichen K 5/08, OTK ZU 9A/2008, Pos. 169.

${ }^{24}$ Urteil des EGMR vom 08.07.2008 in der Sache Vajnai v. Ungarn (Klage Nr. 33629/06).

25 Art. 31 Abs. 3 der Verfassung der Republik Polen: „Einschränkungen, verfassungsrechtliche Freiheiten und Rechte zu genießen, dürfen nur in einem Gesetz beschlossen werden und nur dann, wenn sie in einem demokratischen Staat wegen seiner Sicherheit oder öffentlicher Ordnung oder zum Schutz der Umwelt, Gesundheit, der öffentlichen Moral oder der Freiheiten und Rechte anderer Personen notwendig sind. Diese Einschränkungen dürfen das Wesen der Freiheiten und Rechte nicht verletzen".

26 1) Urteil des Verfassungsgerichtshofs vom 23.04.2008, Aktenzeichen Sk 16/07, OTK ZU 3A/2008, Pos. 45; 2) Urteil des Verfassungsgerichtshofs vom 18.02.2014, Aktenzeichen K 29/12, OTK ZU 2A/2014, Pos. 11.

${ }^{27}$ Der Verfassungsgerichtshof hat sich mehrmals über die Voraussetzungen der Zulässigkeit von Einschränkungen in der Meinungsäußerungsfreiheit ausgesprochen, unter anderem im: 1) Urteil des Verfassungsgerichtshofs vom 22.02.2005, Aktenzeichen K 10/04, OTK ZU 2A/2005, Pos. 17; 2) Urteil des Verfassungsgerichtshofs vom 02.10.2006, Aktenzeichen Sk 34/06, OTK ZU 9A/2006, Pos. 118; 3) Urteil des Verfassungsgerichtshofs K 4/06; 4) Urteil des Verfassungsgerichtshofs vom 17.05.2006, Aktenzeichen K 33/05, OTK ZU 5A/2006, Pos. 57; 5) Urteil des Verfassungsgerichtshofs vom 28.09.2006, Aktenzeichen K 45/04, OTK ZU 8A/2006, Pos. 111. 
nommenen Werte ausdrücken, verurteilt wird, oder gar zum Hass führt ${ }^{28}$. Ohne Zweifel hat dies einen gewissen Einfluss auf die Betrachtungsweise der Meinungsfreiheit, insbesondere dann, wenn Organe der öffentlichen Gewalt zu einer Reaktion gezwungen sind. Dies hat vor allem die Folge, dass der Staat bestimmt, wie der zugelassene Inhalt der Meinungsfreiheit aussieht, und diese Handlung muss nicht unbedingt mit einem demokratischen Staat gleichgesetzt werden. Andererseits müsste man sich darüber Gedanken machen, ob Extremsituationen, in denen von der Meinungsfreiheit Gebrauch gemacht wird, eine Zustimmung verdienen. Die Antwort auf diese Frage ist nicht eindeutig. In der Rechtssprechung des Verfassungsgerichtshofs wird hingegen übereinstimmend angenommen, dass ein Eingriff in die Meinungsfreiheit dann gerechtfertigt ist, wenn dies eine positive Einwirkung auf den Schutz des Gemeinwohls haben sollte, insbesondere im Zusammenhang mit einem angemessenen und wirksamen Handeln der Staatsorgane. Auch der Verfassungsgerichtshof hat festgestellt, dass „die mit der Meinungsäußerungsfreiheit verbundenen Freiheiten und Rechte einer Person keinen absoluten Wert darstellen und mit anderen verfassungsrechtlich geschützten Werten konfrontiert werden müssen, wie etwa mit der öffentlichen Ordnung, deren immananten Bestandteil die ungestörte Tätigkeit der öffentlichen Gewalt darstellt ${ }^{29}$ ". Diese Behauptung muss als gerecht angesehen werden, weil die Autorität im Rahmen einer normalen Funktionsweise des Staates besonders wichtig ist. Sie entscheidet über eine wirksame Ausführung der ihnen übertragenen Aufgaben, die Unterordnung und eine ungestörte Funktionsweise des Staates, und vor allem über das Vertrauen öffentlichen Beamten gegenüber. Die Meinungsfreiheit darf keine bewusste Handlung rechtfertigen, deren Ziel die Beleidigung einer Person darstellt, die eine öffentliche Funktion bekleidet, oder sogar die Erwartung, dass die Gesellschaft es approbiert. Sollte zum Beispiel der Präsident der Republik Polen beleidigt werden, war der Verfassungsgerichtshof der Meinung, dass eine solche Handlung gegen die öffentliche Ruhe und Ordnung verstößt, was eine Voraussetzung der öffentlichen Ordnung darstellt $t^{30}$. In Bezug auf die Tatsache, dass der Präsident der Republik Polen das Staatsoberhaupt ist, bildet er die Verkörperung der staatlichen Würde und Sou-

${ }^{28}$ Als Beispiel kann dienen, wie sich der Leader der Band Behemoth, Adam Darski „Nergal” verhalten hat, als er während eines Konzertes in Gdynia im September 2007 die Bibel zerrissen und die Blätter unter dem Publikum zerstreut hat; siehe: Beschluss des Obersten Gerichts vom 05.03.2015, Aktenzeichen III KK 274/14, LEX Nr. 1734689.

${ }^{29}$ Urteil des Verfassungsgerichtshofs vom 11.10.2006, Aktenzeichen P 3/06, OTK ZU 9A/2006, Pos. 121.

${ }^{30}$ Urteil des Verfassungsgerichtshofs vom 06.07.2011, Aktenzeichen P 12/09, OTK ZU 6A/2011, Pos. 51. 
veränität ${ }^{31}$. Daraus kann also die Schlussfolgerung gezogen werden, dass die Beleidigung des polnischen Präsidenten als ein Angriff auf die Republik Polen betrachtet werden kann. Und weiter bestimmt der Verfassungsgerichtshof, dass ein Angriff auf ein Subjekt, das eine Verkörperung der „Gemeinwohls“ bildet, sich gegen die Republik Polen als das Gemeinwohl aller Bürger richtet, etwa durch eine Herabsetzung des Ansehens der Staatsorgane. An dieser Stelle sollte man sich darüber Gedanken machen, ob eine Einschränkung der MeinungsäuBerungsfreiheit in der öffentlichen Sphäre, z.B. in Bezug auf den Präsidenten der Republik Polen, eine richtige Entscheidung ist. Zusammen mit einer immer weiter reichenden Entwicklung wird die Grenze der Meinungsfreiheit immer weiter verschoben, und Personen, die mit der Tätigkeit öffentlicher Institutionen verbunden sind, müssen sich wegen der von ihnen ausgeführten Aufgaben mit einer weiter reichenden Kritik einverstanden erklären, als Privatpersonen. Die Überschreitung der Grenze der Meinungsäußerungsfreiheit wird aus dem Blickpunkt verschiedener Kriterien beurteilt, zu denen das Niveau der sozialen Akzeptanz oder die Umstände der Überschreitung dieser Grenze gezählt werden können. Nimmt man hingegen eine übermäßige Glorifizierung der verfassungsrechtlichen Position des Präsidenten der Republik Polen an, kommt es zu einer Einschränkung der Meinungsäußerungsfreiheit in der politischen Debatte. In einem demokratischen Staat sollte es zugelassen sein, dass die Handlungen des polnischen Präsidenten unter Kritik gestellt werden, selbst wenn stark kritische Meinungen geäußert werden. Wobei dies nicht bedeutet, dass eine Reaktion seitens der Organe der öffentlichen Gewalt bei der Anwendung bestimmter Mittel des gesetzlichen Schutzes ausgeschlossen wird. Ein anderes Beispiel für die Festlegung der Grenzen der Meinungsäußerungsfreiheit ist die so genannte „Hassrede“, die nach der Beurteilung des Verfassungsgerichtshofs nicht von der Axiologie der demokratischen Rechtssphäre umfasst wird ${ }^{32}$. Dies bezieht sich auf Situationen, deren Gegenstand die Attacke auf bestimmte Gruppen oder Personen wegen deren Rasse, ethnische Herkunft oder Geschlecht ist. In der Literatur wird auch darauf hingedeutet, dass zu dieser Gruppe auch Aussagen zählen, die gegen den so genannten Kanon der politischen Korrektheit verstoßt, zu denen

${ }^{31}$ Siehe: Beschluss des Verfassungsgerichtshofs vom 20.05.2009, Aktenzeichen Kpt 2/08, OTK ZU 5A/2009, Pos. 78.

${ }^{32}$ Im Urteil P 3/06 hat der Verfassungsgerichtshof festgestellt, dass dies unter anderem Inhalte mit einschließt, die Hass wegen der Rasse und Nationalität ausdrücken, eine totalitäre Ideologie propagieren, zur Gewalt aufrufen, um an die Macht zu gelangen, die also gleichzeitig aus der Verfassungsordnung ausgeschlossen sind. 
Negationismus $^{33}$, die Holocaustleugnung, oder eine Belobigung des totalitären Systems $^{34}$ darstellen. Andererseits müsste darüber nachgedacht werden, ob. Die Bekämpfung von Praktiken im Bereich der Hassrede keine Form der Zensur ist? In einem demokratischen Staat sollte man einer (übermäßigen) Zensurkontrolle vorbeugen und einen freien Meinungsaustausch ermöglichen, möglichst maximal ohne einen Eingriff des Staates. Dies schließt aus der öffentlichen Sphäre Anschauungen mit bestimmten Inhalten aus. Die besprochene Institution kann auch zur Ursache deren juristischer Nutzung gegen in Bezug auf eine bestimmte Gruppe „Andersdenkende“ werden. Der Verfassungsgerichtshof hat hingegen in Bezug auf den Schutz der menschlichen Würde festgestellt, dass die Freiheiten und Rechte, die den Inbegriff der menschlichen Würde darstellen, im Falle einer Kollision mit der Meinungsfreiheit das Vorrangsrecht haben können, und deren Einschränkung zur Folge haben ${ }^{35}$. In einer weiteren Entscheidung hat der Verfassungsgerichtshof hingegen anerkannt, dass der ,Schutz der Hochachtung und des guten Rufs, trotz der starken und eindeutigen Verbindung mit der menschlichen Würde, wegen der Notwendigkeit, die Nutzung von Freiheiten und Rechten im erforderlichen Ausmaß durch andere Personen zu garantieren, eingeschränkt werden kann“. Weite wurde festgestellt, dass „diesbezüglich solche Werte, wie Hochachtung, der gute Ruf oder die persönliche Würde (...) nicht mit dem Begriff der in Art. 30 der Verfassung genannten Würde jeder menschlichen Person identifiziert werden können. Die Distinguiertheit hat eine grundlegende Bedeutung für die Festlegung der Grenzen der Meinungsäußerungsfreiheit ${ }^{36 "}$ ". Daraus kann die Schlussfolgerung gezogen werden, dass die Meinungsfreiheit und das Recht auf Schutz der Persönlichkeitsrechte gleichwertig sind, und dass Art. 30 der Verfassung der Republik Polen deren gemeinsame Quelle bildet. Auch die

${ }^{33}$ Der Beauftragte für Bürgerrechte Stelle an den Verfassungsgerichtshof einen Antrag auf Überprüfung der Verfassungsmäßigkeit von Art. 55 des Gesetzes vom 18.12.1998 über das Institut für Nationales Gedenken - die Kommission zur Strafverfolgung von Verbrechen gegen das Polnische Volk (GBl. [Dz. U.] von 2007, Nr. 63, Pos. 424 mit Änderungen) mit Art. 54 Abs. 1 und Art. 73 im Zusammenhang mit Art. 31 Abs. 3 der Verfassung der Republik Polen (Antrag vom 19.09.2008, RPO-595164-I/08/AB). Der im Antrag erwähnte Art. 55 besagt: „Wer öffentlich und den Fakten zuwider Verbrechen, von denen, in Art. 1 Pkt. 1 die Rede ist, leugnet, wird mit einer Buße oder einer Freiheitsstrafe von bis zu 3 Jahren bestraft. Das Urteil wird öffentlich verkundet”. Nach Meinung des Beauftragten für Bürgerrechte überschritt die Annahme dieser Vorschrift die Grenzen der Notwendigkeit in einem demokratischen Rechtsstaat. Siehe: Beschluss des Verfassungsgerichtshofs vom 08.03.2011, Aktenzeichen K 29/08, OTK ZU 2A/2011, Pos. 14.

${ }^{34}$ Konstytucja RP. Tom I. Komentarz art. 1-86, Hrsg. M. Safjan, L. Bosek, Warszawa 2016, S. 1290.

35 Urteil des Verfassungsgerichtshofs, P 10/06.

${ }^{36}$ Urteil des Verfassungsgerichtshofs, Sk 43/05. 
Stellungnahme des Verfassungsgerichtshofs ist erwähnenswert, der die Möglichkeit ausgeschlossen hat, dass der Begriff der Meinungsfreiheit eine uneingeschränkte Beliebigkeit in der persönlichen Verleumdung von Personen, die mit keinen Realien rechnet, umfassen kann ${ }^{37}$. Das Gericht war der Meinung, dass eine Freiheit in diesem Sinne gegen moralische Grundsätze, Gesetzesnormen und demokratische soziale Einrichtungen verstößt, und außerdem nichts mit dem Recht auf Kritik einer beliebigen, auch einer öffentlichen Person zu tun hat.

5. Die Meinungsfreiheit ist eines der Menschenrechte und dient seiner Selbstverwirklichung. Die Möglichkeit einer freien Meinungsäußerung dient also der Entwicklung der Gesellschaft. Es steht außer Frage, dass ohne einen freien Meinungsaustausch keine Chance auf eine ungestörte Funktionsweise eines auf einem demokratischen System basierenden Staates bestehen würde. Die Grundlage dieses Systems bildet gerade die Meinungsäußerungsfreiheit, und sie macht die Toleranz anderen Anschauungen gegenüber erforderlich. Einen besonders hohen Wert hat dies im Rahmen der öffentlichen Debatte, insbesondere in der politischen Sphäre. Die Medien, deren Freiheit einen sozialen Ausmaß hat und eine positive Auswirkung auf die Öffentlichkeit und den Pluralismus des sozialen Lebens haben, spielen hier eine wesentliche Rolle. Dies ist jedoch nicht mit einem komplett uneingeschränkten Recht auf einen freien Meinungsaustausch gleichbedeutend. Die zugelassene Kritik muss sich innerhalb der Grenzen der geltenden gesetzlichen Ordnung befinden. Es ist außerordentlich schwer, die Grenzen der Meinungsäußerungsfreiheit festzulegen; mit diesem Problem haben vor allem Gerichte zu tun, darunter auch der Verfassungsgerichtshof. Die Beurteilung, ob eine Aussage rechtswidrig ist, macht die Berücksichtigung einer Reihe von Faktoren erforderlich, und vor allem der Zeit, in der wir leben. Heute kann eindeutig festgestellt werden, dass sich die Grenze der Meinungsäußerungsfreiheit tatsächlich nach vorne verlegt hat; die Rede ist hier unter anderem von einer spezifischen Brutalisierung der Sprache in der öffentlichen Debatte, sowohl aus dem Blickpunkt der gesetzlichen Vorschriften, aber auch der gesellschaftlichen Normen. In der polnischen Gesellschaft kann bemerkt werden, dass wir es mit einer Liberalisierung der Meinungsfreiheit zu tun haben ${ }^{38}$. Wegen der historischen Hintergründe sieht unsere Gesellschaft, dass eine freie Meinungsäußerung notwendig ist, vor allem sollte jedoch die gegenseitige Toleranz auf den Schild erhoben werden. Damit

${ }^{37}$ Beschluss des Obersten Gerichts vom 22.06.2004, Aktenzeichen V KK 70/04, LEX Nr. 125001.

${ }^{38}$ Siehe: A. Biłgorajski, Granice wolności wypowiedzi czy wolność wypowiedzi ponad granicami? Kilka uwag na temat zakresu wolności wypowiedzi w Rzeczypospolitej Polskiej, [in:] Wolność wypowiedzi i jej granice. Analiza wybranych zagadnień, Hrsg. A. Biłgorajski, Katowice 2014, S. 13. 
entwickelt sich bei uns erst das, was in anderen Ländern bereits zum Standard zählt. Die Aufdeckung der Wahrheit und deren Verbreitung - die Grundlage eines demokratischen Staates bildet das, was als eine Art ,absoluten Wert“ angesehen werden kann. Die Möglichkeit einer Diskussion - insbesondere in der öffentlichen Sphäre - mobilisiert die Gesellschaft und baut deren Stärke auf. Ein Eingriff in diese Sphäre hat Angst und die Vermeidung von Diskussionen aus Angst vor einer Strafe zur Folge, was das soziale Leben demotiviert. Jedem von uns steht die Meinungsäußerungsfreiheit zu, was mehrmals durch den Verfassungsgerichtshof bestätigt worden ist. Es ist also eine Forderung de lege ferenda dem Gesetzgeber gegenüber, dass gesetzliche Vorschriften verabschiedet werden, die sich bezüglich der Eingriffe in die Meinungsäußerungsfreiheit maximal einschränken. Und hier spielen auch die Gerichte eine wesentliche Rolle, denn prinzipiell gestalten diese die Realität und setzen die Grenzen der Meinungsfreiheit; von wesentlicher Bedeutung ist, dass Gerichte rationelle Urteile unter Achtung der Meinungsäußerungsfreiheit sprechen. Für die Zukunft kann die einzige Forderung der Gesellschaft gegenüber sein, dass diese Meinungsäußerungsfreiheit auf eine aggressionsfreie Weise genutzt und diese nicht mit erniedrigenden oder verleumdenden Aussagen gleichgesetzt wird, und dass eine wertvolle öffentliche Debatte nicht durch ein argumentum ad personam ersetzt wird und sich nicht sich auf das Niveau der sozialen Randgruppen beschränkt.

\section{BIBLIOGRAPHY:}

Biłgorajski A., Granice wolności wypowiedzi czy wolność wypowiedzi ponad granicami? Kilka uwag na temat zakresu wolności wypowiedzi w Rzeczypospolitej Polskiej, [in:] Wolność wypowiedzi i jej granice. Analiza wybranych zagadnień, Hrsg. Biłgorajski A., Katowice 2014.

Biłgorajski A., Granice wolności wypowiedzi. Studium konstytucyjne, Warszawa 2013.

Konstytucja RP. Tom I. Komentarz art. 1-86, Hrsg. Safjan M., Bosek L., Warszawa 2016. Piechowiak M., Filozofia praw człowieka. Prawa człowieka w świetle ich międzynarodowej ochrony, Lublin 1999.

Piechowiak M., Prawo naturalne a prawa człowieka, [in:] Substancja. Natura. Prawo naturalne. Zadania współczesnej metafizyki, Hrsg. Maryniarczyk A., Stępień K., Gondek P., Lublin 2006.

Safjan M., Trybunat Konstytucyjny po trzydziestu latach - doświadczenie i przyszłość, Przegląd Konstytucyjny 2017, Nr. 1.

Sobczak J., Wolność ekspresji artystycznej. Standardy europejskie i rzeczywistość polska, [in:] Rada Europy a przemiany demokratyczne w państwach Europy Środkowej $i$ Wschodniej w latach 1989-2009, Hrsg. Jaskiernia J., Toruń 2010. 